\title{
Improving the quality of identification and filtering of micro-object images based on neural networks
}

\author{
Isroil Jumanov $^{1 *}$, Olim Djumanov ${ }^{1}$, and Rustam Safarov ${ }^{1}$ \\ ${ }^{1}$ Department of Information Technologies, Samarkand State University, 140104, University blv. 15, \\ Samarkand, Uzbekistan
}

\begin{abstract}
Constructive approaches, principles, and models for optimizing the identification of micro-objects have been developed based on the use of combined statistical, dynamic models and neural networks with mechanisms for filtering noise and foreign particles of images of medical objects and pollen grains. Algorithms for learning neural networks under conditions of a priori insufficiency, uncertainty of parameters, and low accuracy of data processing are investigated. The mechanisms of contour selection, segmentation, obtaining the boundaries of segments with hard and soft thresholds, filtering using the morphological features of the image have been developed [1]. Mechanisms for recognition and classification of images, adaptation of parameter values, tuning of the network structure, approximation and smoothing of random emissions, bursts in the image contour are proposed. A mechanism for suppressing impulse noise and noise is implemented based on various filtering methods, preserving the boundaries of objects and small-sized parts. Mathematical expressions are obtained for estimating the identification errors caused by nonstationarity, inadequacy of approximation, interpolation, and extrapolation of the image contour. A software package for the recognition and classification of microobjects has been developed. The results were obtained for correct, incorrect recognition, as well as rejected pollen samples, which were synthesized with cubic, biquadratic, interpolation spline-functions and wavelet transforms.
\end{abstract}

\section{Introduction}

The development of scientific and methodological foundations for adaptive processing of information of continuous objects based on the use of neural networks (NN) contribute to solving a wide range of problems related to recognition, classification, approximation, forecasting in control systems of industrial and technological complexes, palynology, environmental protection and ecology, medicine, forensics. In them, the problem of identifying images of micro-objects, in particular, pollen grains, unicellular microorganisms, fingerprints, pictures of useful minerals in the composition of the rock mass, is of great importance [1].

* Corresponding author: rustammix.rs@gmail.com 
This study is devoted to the development of constructive approaches, principles, identification models aimed at using combined statistical, dynamic models and neural networks to optimize the identification of micro-objects with mechanisms for using statistical, dynamic, specific characteristics, as well as filtering non-stationary components of images. It is proved that such methods of image identification have a number of undoubted advantages, simplicity of implementation, high speed of information processing [2].

\section{Main part}

\subsection{Optimization of the identification of micro-objects using the morphological features of images}

At the stage of preprocessing micro-objects, the mechanisms of contour selection, segmentation, obtaining the boundaries of segments with hard and soft thresholds, filtering using the morphological features of the image are implemented. It is supposed to carry out a graphical incision in combination with an image model, determine the intensity of pixels of points inside the segment, outside it, as well as classify images into an object or background [3].

A computational scheme for the identification, recognition, and classification of microobjects, in particular, pollen grains, medical objects, is implemented, which includes the following blocks: input image input, preprocessing, identification of objects of interest, highlighting features and image features, recognition, and classification of a micro-object, presentation of results. The effectiveness of the proposed computational scheme is based on real data from the chest X-ray processing (CXR) system [4, 5, 13-15].

It was determined that images of medical objects have noticeable noise, different levels of contrast and require the use of filtering mechanisms to eliminate "noise", "interference", "foreign microparticles".

The study began with the use of a median filter as a basic mechanism for combating Gaussian and Poisson noise, which performs the functions of enhancing contrast, defining and shaping segment boundaries, and classifying images. Filtering mechanisms improve the definition of image parameters, in particular, signal/noise ratio, visual appearance, removes noise and unwanted parts.

For image classification, it is proposed to use a competing probabilistic neural network $(\mathrm{CPNN})$, which is insensitive to signal/noise deviations and generates parameter values with an accurate estimate of the probability. CPNN forms the level of competition between Gaussian kernels and conditional probabilities.

Application of the mechanism is associated with ranking the value of the conditional probability in descending order. All cores compete to be assigned to the appropriate class. In this case, only significant values of conditional probabilities are used. The points are approximated by averaging multidimensional normal Gaussian kernels, forming the product of their own one-dimensional kernels, centering them in each sample according to the Gaussian distribution $[5,6]$

$$
W_{i, j}=\frac{1}{(2 \pi)^{\frac{d}{2}} \sigma^{d}} \cdot \exp \left(-\frac{x_{n e w}-x_{C i, j}}{2 \sigma^{2}}\right),
$$

where $|C|_{i}$ - cardinality of subset $C_{i} ; W_{i, j}-$ multidimensional normal kernel; $\sigma$ - spread, which is an adjustable parameter; $X_{C i, j}-1$ - input of the classifier; $N C$ - set of input images to be classified $C_{i}=\left\{X_{C_{i, 1}}, X_{C_{i, 2}}, \ldots, X_{C_{i,\left|C_{i}\right|}}\right\}$.

The number of selected cores in class $i$ determines the number of winners $N W_{i}$. 
The ratio of $N W_{i}$ to the total number of cores in each class $i$ is given as $\gamma_{i}=\frac{N W_{i}}{\left|C_{i}\right|} \rightarrow N W_{i}=\left[\gamma_{i}\left|C_{i}\right|\right]$, where $\gamma_{i \min }=\frac{1}{\left|C_{i}\right|} \leq \gamma_{i} \leq 1$ - continuous variable.

The conditional probability of each class is calculated as $P_{i}=\frac{1}{N W_{i}} \sum_{i=1}^{N W_{i}} w_{i j}=\frac{1}{\left[\gamma_{i}\left|C_{i}\right|\right]} \sum_{i=1}^{\left[\gamma_{i}\left|C_{i}\right|\right]_{w_{i j}}}$.

The traditional approach considers when $\gamma_{i}=1$.

The efficiency of the mechanism depends on parameters $\sigma$ and $\gamma_{i}$, which contribute to the optimization of the classification based on control operators: expanding and contracting the size of the training set to achieve maximum performance. A two-level optimization has been implemented, in which $\sigma$ is considered at the top level, and $\gamma_{i}$ at the bottom level. The top-level optimization assumes that all values of $\gamma_{i}$ remain constant and equal to one. At the lower level, optimum $\sigma$ is achieved by adjusting the value of parameter $\gamma_{i}$.

When classifying on the basis of CPNN, the values of all nuclei are sorted in descending order, a new class 2 is formed, corresponding to $P_{1}^{C P N N}<P_{2}^{C P N N}$.

\subsection{Optimization of the identification of micro-objects based on the CPNN adaptive learning mechanism}

Dynamic neural networks are investigated. In this case, the mechanism of classification of micro-objects is performed in the following stages: input of new images $x_{\text {new }}$; calculate the Gaussian kernel for each input vector; sorting kernels in each class, forming them in descending order:

$$
\begin{aligned}
& \left\{\omega_{1,1}, \omega_{1,2}, \omega_{1,3}, \ldots . . \omega_{1,\left|C_{1}\right|}\right\}=\left\{\omega_{1,1}^{\prime}, \omega_{1,2}^{\prime}, \omega_{1,3}^{\prime}, \ldots . . \omega_{1,\left|C_{1}\right|}^{\prime}\right\} \\
& \left\{\omega_{2,1}, \omega_{2,2}, \omega_{2,3}, \ldots . . \omega_{2,\left|C_{1}\right|}\right\}=\left\{\omega_{2,1}^{\prime}, \omega_{2,2}^{\prime}, \omega_{2,3}^{\prime}, \ldots . . \omega_{2,\left|C_{1}\right|}^{\prime}\right\} \\
& \left\{\omega_{N C, 1}, \omega_{N C, 2}, \omega_{N C, 3}, \ldots . . \omega_{N C,\left|C_{N C}\right|}\right\}=\omega_{N C, 1}^{\prime}, \omega_{N C, 2}^{\prime}, \omega_{N C, 3}^{\prime}, \ldots . . \omega_{N C,\left|C_{N C}\right|}^{\prime}
\end{aligned}
$$

Further, from the sorted nuclei in class $I$, the $N W_{i}$ nucleus with the highest rating $N W_{i}=\left[\gamma_{i} \mid C_{i}\right]$ is selected. Instead of the used kernels, the selected kernels in the last step are used.

The conditional probability of classification is calculated for each class

$$
\begin{aligned}
& \left\{\omega_{1,1}^{\prime}, \ldots \ldots, \omega_{1, N W_{1}}^{\prime}\right\} \rightarrow P_{1}=\left(N W_{1}\right)^{-1} \sum_{j=1}^{N W_{1}} \omega_{1, j}^{\prime} ; \\
& \left\{\omega_{2,1}^{\prime}, \ldots \ldots, \omega_{2, N W_{2}}^{\prime}\right\} \rightarrow P_{2}=\left(N W_{2}\right)^{-1} \sum_{j=1}^{N W_{2}} \omega_{2, j}^{\prime} ; \\
& \left\{\omega_{N C, 1}^{\prime}, \ldots \ldots, \omega_{N C, N W_{N C}}^{\prime}\right\} \rightarrow P_{N C}=\left(N W_{N C}\right)^{-1} \sum_{j=1}^{N W_{N C}} \omega_{N C, j}^{\prime} .
\end{aligned}
$$

A class with a higher conditional probability is selected and assigned to the new input class $x_{\text {new }}=\arg \max \left\{P_{i}\right\}, 1 \leq i \leq N C$.

Implementation of the adaptive learning mechanism of CPNN was tested on the basis of a set of 80 images of objects with lung pathology and 50 images without their pathology. Recognition of medical objects was assessed by the coefficients of similarity $\Omega$, similarity by mean value, distance to the contour of the object $[7,14]$.

It is determined that the metrics mainly depend on the following values $T P, T N, F P, F N$ and $R N_{C n t}$, where $T P$ - the number of pixels of the correctly identified area; $T N$ - the number of pixels, correctly detected area as background; $F P$ - number of 


\section{ICECAE 2021}

pixels, falsely identified area; $F N$ - number of pixels, falsely detected area as background; $R N_{C n t}$ - the number of reference pixels of the edges on the outline of the image.

The similarity rate is defined as $\Omega=\frac{T P}{T P+F P+F N}$. The mean similarity coefficient is defined as

$$
D=\frac{2 \cdot T P}{2 \cdot T P+F P+F N} .
$$

Metrics are set with values from 0 to 1 . When the metric value is 1 , then a fully overlapping segmentation is applied. The classification value of each pixel has the same effect on the result of calculating the distance to the boundary of the contour. The reliability of the metric assessment is made by the distance to the boundary of the reference sample contour. The minimum distance of each point on the border $S$ to the contour $R$ is determined.

Let $S_{i}, i=1, \ldots, n_{S}$ and $R_{j}, j=1, \ldots, n_{R}$ be points on the detected boundary of the object $S$ and the reference boundary of the reference object $R$. The minimum distance of point $S_{i}$ to $R$ is defined as $d\left(S_{i}, R\right)=\min _{j}\left\|r_{j}-s_{i}\right\|$ with a factor of

$$
A(S, R)=\frac{1}{2}\left(\frac{\sum_{i} d\left(s_{i}, R\right)}{n_{S}}+\frac{\sum_{j} d\left(r_{j}, R\right)}{n_{R}}\right) .
$$

Table 1 shows quantitative assessments of the effectiveness of the learning mechanism of CPNN based on the identified boundaries of the lungs $[8,9]$.

Table 1. The effectiveness of the CPNN learning mechanism.

\begin{tabular}{|c|c|c|c|c|}
\hline \multicolumn{5}{|c|}{ Statistical characteristics of the mechanism } \\
\hline Coefficients & Minimum $a$ & $a \pm 3 \sigma$ & Middle $a$ & Maximum $a$ \\
\hline Similarities & 0.952 & $0.969 \pm 0.016$ & 0.968 & 0.985 \\
\hline Middle & 0.965 & $0.964 \pm 0.012$ & 0.967 & 0.990 \\
\hline Distance to object outline & 0.957 & $0.975 \pm 0.015$ & 0.970 & 0.989 \\
\hline Average distance & 1.247 & $1.642 \pm 0.323$ & 1.523 & 2.551 \\
\hline
\end{tabular}

Object classification accuracy was investigated on the basis of joint training sets MC - 40 CXR images with pathologies and SH - 40 CXR images without pathology. It has been determined that the average accuracy of object classification is $96 \%$. Greater classification accuracy is achieved with a set of SH than MC. The classification error for CXR images is about 4-5\%. The research results are shown in table 2.

Table 2. Comparative assessments of the effectiveness of the CPNN learning mechanism.

\begin{tabular}{|c|c|c|c|c|c|c|}
\hline \multirow{2}{*}{ Images } & \multicolumn{3}{|c|}{ MC - set } & \multicolumn{3}{c|}{ SH - set } \\
\cline { 3 - 4 } \cline { 6 - 7 } & Number & \multicolumn{2}{|c|}{ Classified \% } & \multirow{2}{*}{ Classified \% } \\
\cline { 3 - 4 } \cline { 6 - 7 } & Correctly & Incorrectly & & Correctly & Incorrectly \\
\hline $\begin{array}{c}\text { without } \\
\text { pathology }\end{array}$ & 75 & 96.94 & 3.06 & 99 & 97.89 & 2.11 \\
\hline $\begin{array}{c}\text { with } \\
\text { pathology }\end{array}$ & 50 & 96.58 & 3.42 & 80 & 95.77 & 4.23 \\
\hline $\begin{array}{c}\text { Total } \\
\text { number }\end{array}$ & 125 & 96.76 & 3.24 & 179 & 96.83 & 3.17 \\
\hline
\end{tabular}


Table 3 shows the result of a comparative analysis of the efficiency of the filtering mechanism for images of micro-objects.

Table 3. Effectiveness of image filtering mechanisms.

\begin{tabular}{|c|c|}
\hline Filters & Distance to object outline \\
\hline Prewitt & 24.68 \\
\hline Sobel & 26.25 \\
\hline Shear and wavelet transform & 27.05 \\
\hline Backshifted and wavelet transform & 33.50 \\
\hline CPNN & 34.36 \\
\hline
\end{tabular}

\subsection{Optimization of micro-objects identification based on adaptive image filtering mechanisms}

A modified mechanism of vector median filtering (VMF) is proposed, which allows suppressing the noise part, interference in images. The mechanism ensures the preservation of the most important features, properties of objects that are used in detection, selection, segmentation $[10,11,12,15]$. When filtering images, the mechanism receives new values of the points of the boundaries of the contour of each component, selects windows for points by values of the median in the form

$$
\begin{aligned}
& C_{m}\left(R_{m}, G_{m}, B_{m}\right) ; \quad\left(R_{0}, R_{1}, \ldots, R_{p-1}, R_{m}\right), R_{0} \leq R_{1} \leq \ldots \leq R_{p-1} ; m=p / 2 ; \\
& \left(G_{0}, G_{1}, \ldots, G_{p-1}, G_{m}\right), G_{0} \leq G_{1} \leq \ldots \leq G_{p-1} ; m=p / 2 ; \\
& \left(B_{0}, B_{1}, \ldots, B_{p-1}, B_{m}\right), B_{0} \leq B_{1} \leq \ldots \leq B_{p-1} ; m=p / 2 ;
\end{aligned}
$$

The characteristics of brightness, color coding in the coordinate space of the components of the original images, which are set by seven discrete values: $0,169,77,104,40,122,125$, are investigated. $5 \times 5$ filtering is performed for each tone component, which is set by 16 discrete values: $0,169,77,104,122,125,126,114,115,137,109,45,46,105,35,32$. The mechanism preserves the boundaries of objects, small-sized details, color characteristics of the image.

The vector median filter with the minimum distance from the middle vector $C_{\text {mid }}\left(R_{\text {mid }}, G_{\text {mid }}, B_{\text {mid }}\right)$ is determined by $R_{\text {mid }}=\frac{1}{p} \sum_{i=0}^{p-1} R_{i}, p$ is the total number of elements in the window. The distance $d$ from each window element to the vector $C_{m i d}$ is located in non-decreasing order $d_{0} \leq d_{1} \leq \ldots \leq d_{p-1}$. This approach does not ensure the preservation of the boundaries of classification objects.

Preservation of information about borders and textures is achieved due to the fact that from all components of the set of images the one in which the variance is minimal is selected.

Then the filter has the vector of the median of the component with the minimum variance, spaced from the vector $C_{m}$ at the minimum distance $\min _{k \in\left[1, N_{w}\right]} \varepsilon_{w k}=\sum_{j \in\left[1, N_{w}\right]}\left\|C_{j}-C_{m k}\right\|, k$ is the number of the set component, $w$ is the filtering window number, $N_{w}$ is the number of components in the $i$-th window of the set, $p_{w}$ is the aperture size, $C_{m k}$ is the estimate $C_{m}$ in the $k$-th aperture.

Two sizes are selected for each window. The first, with the minimum variance, and the second with the estimate of the median $C_{m k}\left(R_{m k}, G_{m k}, B_{m k}\right)$.

A comparison is made of the distance between the estimates for the threshold values $T=\left(R_{t}, G_{t}, B_{t}\right)$. In this case, if the distance is greater than it, then the filtering mechanism is 
selected from a larger aperture $W_{2}$, which is at a minimum distance from the component median. If the distance between the medians is less than the threshold, then the filtering mechanism is selected from the smaller aperture $W_{1}$. If this distance is less than the threshold, then the output element of the filter is the central element of the aperture. As threshold value, $T$ the mean square deviation of the "additive noise" of the image is selected

$$
C_{\text {out }}=\left\{\begin{array}{l}
\arg \min _{C_{i} \in W_{2}}\left\|C_{i}-C_{m 2}\right\|,\left\|C_{m 1}-C_{m 2}\right\|>T ; \\
C_{c}, \quad\left\|C_{c}-C_{m 1}\right\| \leq T ; \\
\arg \min _{C_{i} \in W_{1}}\left\|C_{i}-C_{m 1}\right\|,\left\|C_{m 1}-C_{m 1}\right\| \geq T,
\end{array}\right.
$$

where $C_{c}$ - center aperture element, $C_{\text {out }}$ - filter outlet element, $C_{m 1}$ - median calculated in the smaller aperture, $C_{m 2}$ - median calculated in a larger aperture.

It is determined that the adaptive filtering mechanism provides better preservation of boundaries, uses the estimate of the median along the boundary of the object. Small detail suppression is determined by the size of the smaller window aperture. Impulse noise suppression is performed by median filtering in a larger window.

\subsection{Optimization of identification of micro-objects based on the mechanism of filtering noise and impulse noise in the image}

Modeling noise, impulse interference is performed as follows. The realizations of a random process distributed according to a normal law with statistical parameters $\left(0, \sigma_{\mathrm{n}}\right)$ are studied as noise. It is assumed that the noise $n(l, m)$ is superimposed on each component of the image.

Pulse hindrance $I$ is modeled by pseudo-random values, the coordinates of the samples, which are selected according to a uniform distribution law. The numbers of counts affected by noise and impulse hindrance were determined by the probability of $P$.

Image sample signals are modeled by the equation

$$
C(l, m)=\left\{\begin{array}{lc}
S(l, m)+n(l, m), & (1-P), \\
I(l, m), & P,
\end{array}\right.
$$

where $S(l, m)$ - image samples.

The mean square deviation (MSD) criterion evaluates the filtering quality of the samples of the image components as $\sigma=\left({\sigma_{R}}^{2}+{\sigma_{G}}^{2}+{\sigma_{B}}^{2}\right)^{1 / 2}$.

Evaluated in the case of using eight-bit quantization, the value of the peak signal-to-noise ratio is calculated by the $\psi=201 \mathrm{~g}(255 / \sigma)$.

It was determined that the smallest image identification error is achieved with vector median filtering (VMF) and cascading multichannel median filter (CMMF).

The characteristics of identification of filtration mechanisms synthesized with NS were investigated, the results of which are shown in table 4.

The CMMF mechanism provides more efficient filtering, which also provides better preservation of information about the high frequencies of the image.

Estimates of filtering errors for the considered mechanisms are obtained on ideal images, as well as on images containing additive noise and impulse noise, confirming the effectiveness of the mechanism.

The developed mechanism is implemented in the form of functional modules of the software package (SP) for visualization, recognition, and classification of pollen grains.

Table 4. Efficiency of vector median filtering based on NN synthesis. 


\begin{tabular}{|c|c|c|c|c|c|}
\hline Median filtering & $\sigma_{R}$ & $\sigma_{G}$ & $\sigma_{B}$ & $\sigma$ & $\psi$ \\
\hline CMMF & 1,05 & 1,47 & 1,55 & 2,38 & 40,6 \\
\hline AMF $3 \times 3$ & 2,23 & 3,33 & 4,37 & 5,9 & 32,67 \\
\hline M $3 \times 1$ & 2,88 & 3,8 & 4,04 & 6,24 & 32,2 \\
\hline M $5 \times 1$ & 3,96 & 5,3 & 5,53 & 8,62 & 29,4 \\
\hline MF $3 \times 3$ & 6,82 & 9,15 & 8,8 & 14,41 & 24,95 \\
\hline MF $5 \times 5$ & 7,52 & 10,25 & 9,67 & 15,97 & 24,1 \\
\hline
\end{tabular}

Table 5 shows the effectiveness of CMMF in eliminating additive image noise.

Table 5. The efficiency of CMMF in eliminating additive noise.

\begin{tabular}{|c|c|c|c|c|c|}
\hline \multirow{2}{*}{ Quantization level } & \multicolumn{5}{|c|}{$\sigma$ additive normal noise } \\
\cline { 2 - 6 } & $\sigma_{R}$ & $\sigma_{G}$ & $\sigma_{B}$ & $\sigma$ & $\psi$ \\
\hline 5 & 1,28 & 1,68 & 1,81 & 2,78 & 39,2 \\
\hline 10 & 3,31 & 3,55 & 3,81 & 6,17 & 32,3 \\
\hline 15 & 7,22 & 7,35 & 7,74 & 12,89 & 25,9 \\
\hline 20 & 11,14 & 11,37 & 11,79 & 19,8 & 22,2 \\
\hline
\end{tabular}

Two types of experimental research were carried out in the system of selection and seed production, for example, modeling of images of pollen grains. In the first, images of pollen grain samples are formed, analyzed, pixels of images are determined, i.e. "Pollen spots" that differ from background pixels after segmentation of the original image. These pixels are classified as "black" or "white". In the first experiment, "pollen" or "non-pollen" image windows are formed. The pollen-spot segmentation model distinguishes pollen pixels from pixels in the background of an image. In a second experiment, the segmentation model is applied to a new set of windows that are used to classify pollen species. The image model is trained based on a set of 7,007,000,000 pixels.

As a result, an average of $9 \%(637,000,000)$ pixels are labeled as "not pollen".

Estimates of the effectiveness of the mechanism with other filtration methods are given in the table 6 .

Table 6. Evaluation of the effectiveness of the filtering mechanism based on NN.

\begin{tabular}{|l|l|r|r|r|c|}
\hline Hindrance & CMMF & MF $3 \times 3$ & MF $5 \times 5$ & AMF $3 \times 1$ & AMF $5 \times 1$ \\
\hline \multirow{4}{*}{ No noise } & 0,39 & 18,74 & 20,09 & 0,78 & 3,74 \\
\cline { 2 - 6 } & 0,27 & 15,27 & 16,18 & 0,48 & 4,43 \\
\cline { 2 - 6 } & 0,42 & 15,95 & 16,79 & 0,46 & 4,61 \\
\cline { 2 - 6 } & 0,63 & 28,96 & 30,78 & 1,02 & 7,41 \\
\hline \multirow{4}{*}{ Normal (0,100) } & 9,70 & 19,13 & 21,28 & 7,11 & 7,58 \\
\cline { 2 - 6 } & 9,76 & 15,02 & 15,98 & 7,1 & 7,29 \\
\cline { 2 - 6 } & 9,71 & 15,74 & 16,86 & 7,1 & 7,41 \\
\cline { 2 - 6 } & 16,84 & 28,97 & 31,5 & 12,3 & 12,86 \\
\cline { 2 - 6 } & 2,72 & 18,46 & 20,22 & 6,82 & 3,99 \\
\cline { 2 - 6 } & 2,44 & 15,07 & 16,29 & 6,82 & 4,57 \\
\cline { 2 - 6 } & 2,21 & 15,72 & 16,9 & 6,91 & 4,87 \\
\hline \multirow{3}{*}{$\begin{array}{l}\text { Additive (normal } \\
\text { pulsed) }\end{array}$} & 4,27 & 28,54 & 30,98 & 11,86 & 7,78 \\
\cline { 2 - 6 } & 1,06 & 19,94 & 20,74 & 7,34 & 7,68 \\
\cline { 2 - 6 } & 10,03 & 15,68 & 15,52 & 7,4 & 7,4 \\
\cline { 2 - 6 } & 9,97 & 16,4 & 16,41 & 7,52 & 7,54 \\
\hline \multirow{2}{*}{ Pulsed } & 17,36 & 30,2 & 30,66 & 12,85 & 13,06 \\
\hline
\end{tabular}


A new mechanism for training an image model based on a hash code has been proposed and implemented, which allows calculating a numerical characteristic, establishing the indices of each pixel, characteristics of its neighborhood. Each hash code indicates the "shape class" or category to which the pixel belongs, is an integer in the range from 1 to $N, N$ is the parameter to be optimized. The number of different types of pollen to be recognized is controlled. The more training examples are used, the more the model is justified.

The hash-based learning mechanism is presented as a program module, which includes the following operators: identifying pixels, surroundings of the pixel to be classified; adapting the size of 64-bit hash codes, modular division range. The dimensions of the mesh $2 \times 2,4 \times 4,8 \times 8,16 \times 16,32 \times 32,64 \times 64$ and $128 \times 128$. For example, $8 \times 8$ grids with 1 -pixel spacing means that all 64 neighboring pixels are counted. $8 \times 8$ grids with 2 -pixel spacing mean every other pixel will be counted.

To reduce the dimension of the input space, hash codes are calculated, the results are generated in the database for both positive and negative examples. If there are more positive results than negative results, then the candidate pixel is classified as pollen. Otherwise, as a negative example, that is, as a slide background.

\section{Conclusion}

Scientific and methodological foundations for optimization of identification of micro-objects using threshold segmentation mechanisms that use various filtering and discrete wavelet transforms have been developed. It has been determined that the developed mechanisms can improve the accuracy of segmentation, detection of boundaries of objects of interest by $1-2 \%$ compared to traditional technologies for determining the boundaries of objects in CXR images. To determine the contours of objects of interest, the mechanism of vector median filtering is implemented, combined with a shift wavelet transform.

A computational scheme for the identification of CXR images based on the CPNN adaptive learning mechanism has been proposed, which has been tested for determining the boundaries of the lungs and recognizing chest pathologies, including the detection of tumors on CT images. The average accuracy of object (pathology) recognition reaches $96 \%$.

A mechanism for recognizing micro-objects has been developed, which includes the competition operator, calculating Gaussian kernels and conditional probabilities, the effectiveness of which is clearly manifested on the basis of $\mathrm{NN}$.

A mechanism for suppressing impulse noise and noise has been implemented based on the use of CMMF with VMF, which ensures the preservation of the boundaries of objects and small-sized parts. The maximum efficiency of CMMF is manifested when eliminating impulse noise.

\section{References}

1. R. Rendondo, V. Marcos Computers and Electronics in Agriculture Pollen segmentation and feature evaluation for automatic classification in bright-field microscopy 110 pp 56-69 (2014)

2. M. Chica Computer Science Standard methods for inexpensive pollen loads authentication by means of computer vision and machine learning URL: https://arxiv.org/abs/1511.04320, (2015)

3. M. Chica, P. Campoy Journal of Food Engineering Discernment of bee pollen loads using computer vision and oneclass classification techniques 112 pp 50-59, (2012)

4. I.I. Jumanov, O.I. Djumanov, R.A. Safarov Chemical Technology, Control and Management Optimization of image processing using characteristics and peculiarities of pollen grains 5 pp 71-78 (2019) 
5. I.I. Jumanov, O.I. Djumanov, R.A. Safarov Int. Russian Automation Conf. Optimization of Identification of Images of Micro-Objects Taking Into Account Systematic Error Based on Neural Networks pp. 626-631 (2020)

6. N. Khanzhina, E.B. Zamyatina Perm University Press (Perm) Using classical methods and neural networks for pollen grain recognition vol 4(23) pp 111-119 (2013)

7. A. S. Chernykh, E.B. Zamyatina Perm State Nat. Research University Study of the possibility of using a number of classical methods for the recognition of pollen grain $\mathrm{s}$ pp 161-169 (2012)

8. D.A. Tarkhov Neural networks. Models and algorithms D.A.Tarkhov Moscow Radio engineering 18 pp 255 (2005)

9. I.I. Jumanov Problems of Informatics and Energy Optimization of image processing of micro-objects based on recurrent learning of a neural network and implicative selection of informative features 4 pp 12 (2016)

10. O.I. Djumanov SibSUTI Press (Novosibirsk) Adaptive neural network system for image visualization, recognition and classification of micro-objects 2 pp 76-86 (2008)

11. I.I. Jumanov Bulletin of TUIT (Tashkent) Optimization of data processing of nonstationary objects based on fuzzy identification models with setting parameters 1(41) pp 34-47 (2017)

12. O.I. Djumanov X int. scientific method. conf.( Rostov-on-Don) A neural network system for adaptive information processing of a non-stationary nature in the management of a university 6 pp 81-85 (2008)

13. I.I. Jumanov, O.I. Djumanov, R.A. Safarov 11 th World Conference "Intelligent System for Industrial Automation" (WCIS-2020) 1323 pp 170-179 (2020) DOI:10.1007/9783-030-68004-6_22

14. Ibragimovich, J.I., Isroilovich, D.O., Abdullayevich, S.R. 2020 International Conference on Information Science and Communications Technologies 9351483 (2020) DOI: 10.1109/ICISCT50599.2020.9351483

15. I.I. Jumanov, O.I. Djumanov, R.A. Safarov Journal of Physics: Conference Series, 1791(1), 012099 (2021) DOI:10.1088/1742-6596/1791/1/012099 